\title{
From Quark Stars to Hybrid Stars
}

\author{
Alessandro Drago $^{a}$ and Andrea Lavagno ${ }^{b}$ \\ ${ }^{a}$ Dipartimento di Fisica, Università di Ferrara and INFN, Sezione di Ferrara, \\ 44100 Ferrara, Italy \\ ${ }^{b}$ Dipartimento di Fisica, Politecnico di Torino and INFN, Sezione di Torino, \\ 10129 Torino, Italy
}

\begin{abstract}
We show the possible existence of compact stars having a surface composed of a mixed phase of quarks and hadrons. This scenario can be realized both for self-bound stars, satisfying the so-called Witten-Bodmer hypothesis, and for gravitationally bound stars. This class of solutions of the Tolman-Oppenheimer-Volkoff equation can be obtained in all the models we discuss, within a physically acceptable range of values of the model parameters.
\end{abstract}

PACS: 26.60.+c, 12.38.Mh, 97.60.Jd

Keywords: Neutron stars; Quark matter; Phase transition

Recently several analysis of observational data have emphasized the possible existence of compact stars having very small radii, of the order of 9 kilometers or less [1]. To account theoretically for the existence of such a star, one would need to use of very soft Equations of State (EOSs), that can be obtained, in principle, introducing kaon condensation [2-4], or hyperonic degrees of freedom [5-7]. Another possibility is to consider quark stars, where the density of the surface is above nuclear matter saturation density. These objects have been obtained till now assuming the validity of the hypothesis introduced independently by Bodmer [8] and Witten [9], which states that the true and absolute ground state of the strong interaction is a state of deconfined quark matter. The latter consists of an approximately equal number of up, down and strange quarks having energy per baryon $E / A$ smaller than that of iron $\left(E_{F e} \approx 930 \mathrm{MeV}\right)$, at zero temperature and pressure [10]. Stars entirely composed of matter in such ultra-stable state are self-bound [11,12] and could rotate with a period well below one millisecond $[13,14]$.

A possible problem in interpreting neutron stars as strange quark stars is the difficulty of having glitches in the latter, since the most widely accepted model for the glitches require the existence of a crystallized phase trapping magnetic 
flux tubes. It is not clear whether such a structure can be obtained in quark stars, although the presence of a color superconductive phase could open this possibility [15].

The aim of this letter is to show that it is possible to find a minimum of the energy per baryon number at finite density, in the mixed quark-hadron phase. This minimum corresponds to an energy which can be either above or below $E_{F e}$. Stars builded using this EOS will be gravitationally bound, if the minimum is above $E_{F e}$, or self-bound stars if the minimum is below $E_{F e}$. In both cases the surface of the star is made of mixed quark-hadron phase. To our knowledge, this possibility has never been investigated, because in general the so-called quarks stars have been studied using the quark EOS alone, without matching it with the hadronic EOS. In this paper, on the contrary, we will study in detail the transition from a low density EOS (hadrons) to a high density one (quarks), using the Gibbs conditions.

The EOS appropriate to the description of a compact star has to satisfy $\beta$ stability conditions. This implies the existence of two conserved charges, the baryonic charge and the electric charge. When the Gibbs conditions are applied in presence of more than a single conserved charge, the technique developed by Glendenning has to be adopted [16-18]. Using that technique, the pressure need not to be constant in the mixed phase, what will be crucial in our investigation.

The conservation of the baryon (B) and the electric (C) charge can be written as follows:

$$
\begin{aligned}
& \rho_{B}=(1-\chi) \rho_{B}^{h}+\chi \rho_{B}^{q} \\
& \rho_{C}=(1-\chi) \rho_{C}^{h}+\chi \rho_{C}^{q}+\rho_{e}+\rho_{\mu}=0 .
\end{aligned}
$$

Here $\chi$ is the fraction of matter in the quark phase. The superscripts $h$ and $q$ label the density in the hadronic and in the quark phase, respectively. The electron $\left(\rho_{e}\right)$ and the muon $\left(\rho_{\mu}\right)$ charge densities contribute to make the total electric charge equal to zero.

The equations of chemical equilibrium under $\beta$-decay and deconfinement are the following:

$$
\begin{gathered}
\mu_{n}-\mu_{p}=\mu_{e} \quad, \quad \mu_{n}-\mu_{p}=\mu_{\mu} \\
2 \mu_{d}+\mu_{u}=\mu_{n} \quad, \quad \mu_{u}-\mu_{d}=\mu_{p}-\mu_{n} \\
\mu_{s}=\mu_{d} .
\end{gathered}
$$

The usual condition for mechanical equilibrium, i.e. the equality of the pres- 
sure in the two phases, reads:

$$
P^{h}=P^{q}
$$

The previous equations have to be solved together with the field's equations for the adopted hadronic and quark models. Concerning the hadronic phase, we have used the relativistic non-linear Walecka-type models of GlendenningMoszkowsky (GM1, GM2, GM3) [5] and Bodmer (B91) [19]. For the quark phase we have considered the MIT bag model at first order in the strong coupling constant $\alpha_{s}[10]$ and the Color Dielectric Model (CDM) [20,21]. In the latter, quarks develop a density dependent constituent mass through their interaction with a scalar field $\kappa$ representing a multi-gluon state. The Lagrangian of the CDM reads:

$$
\begin{aligned}
L & =i \bar{\psi} \gamma^{\mu} \partial_{\mu} \psi+\frac{1}{2}\left(\partial_{\mu} \sigma\right)^{2}+\frac{1}{2}\left(\partial_{\mu} \vec{\pi}\right)^{2}-U(\sigma, \vec{\pi}) \\
& +\sum_{f=u, d} \frac{g_{f}}{f_{\pi} \kappa} \bar{\psi}_{f}\left(\sigma+i \gamma_{5} \vec{\tau} \cdot \vec{\pi}\right) \psi_{f}+\frac{g_{s}}{\kappa} \bar{\psi}_{s} \psi_{s} \\
& +\frac{1}{2}\left(\partial_{\mu} \kappa\right)^{2}-\frac{1}{2} M^{2} \kappa^{2}
\end{aligned}
$$

where $U(\sigma, \vec{\pi})$ is the "mexican-hat" potential, as in Ref. [22].

The coupling constants are given by $g_{u, d}=g\left(f_{\pi} \pm \xi_{3}\right)$ and $g_{s}=g\left(2 f_{K}-f_{\pi}\right)$, where $f_{\pi}=93 \mathrm{MeV}$ and $f_{K}=113 \mathrm{MeV}$ are the pion and the kaon decay constants, respectively, and $\xi_{3}=f_{K^{ \pm}}-f_{K^{0}}=-0.75 \mathrm{MeV}$. These coupling constants depend on a single parameter $g$. Confinement is obtained via the effective quark masses $m_{u, d}=-g_{u, d} \bar{\sigma} /\left(\bar{\kappa} f_{\pi}\right)$ and $m_{s}=g_{s} / \bar{\kappa}$, which diverge outside the nucleon. Working at mean-field level, the only free parameter is actually the product $G=\sqrt{g M}$. In this model the last term of the lagrangian plays a role similar to the vacuum pressure constant $B$ of the MIT bag model. At variance with the latter, in the CDM the vacuum pressure is density dependent.

Let us discuss our results about the EOS in the mixed hadron-quark phase applying the above discussed Gibbs conditions. In Fig. 1, we report the pressure as a function of the baryon density for different values of the MIT-bag constant $B^{1 / 4}[\mathrm{MeV}]$, with $\alpha_{s}=0, m_{u}=m_{d}=0, m_{s}=100 \mathrm{MeV}$ and the GM3 hadronic model [5]. The figure shows that decreasing the bag constant from the value $B^{1 / 4}=170 \mathrm{MeV}$, corresponding to a typical hybrid star EOS (composed by a central quark phase, an intermediate mixed phase and an hadronic crust at low density), to the value $B^{1 / 4}=158 \mathrm{MeV}$, corresponding to a pure quark star (with a nonzero density at the surface), we have a region where the pressure decreases by increasing the baryon density (instability condition) and, for some values of $B^{1 / 4}$, we have an EOS which exhibits a double 


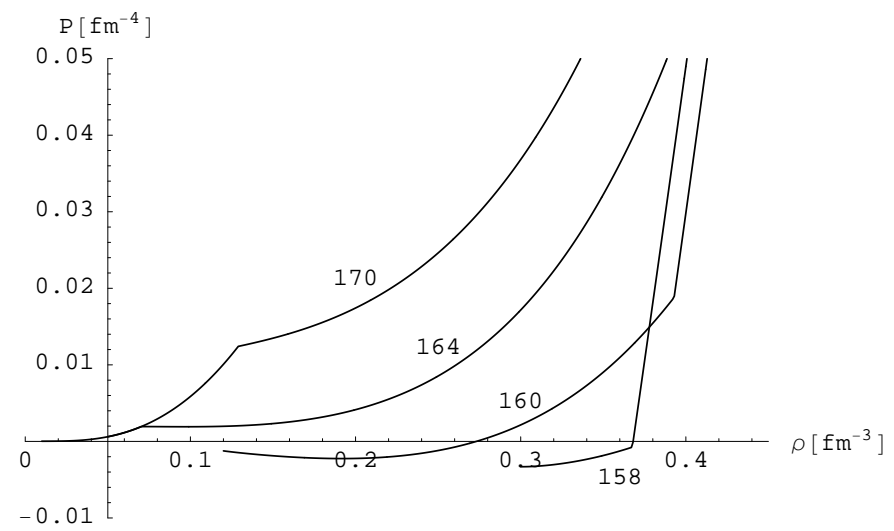

Fig. 1. The pressure as a function of the baryon density for various values of the MIT-bag constant $B^{1 / 4}[\mathrm{MeV}]$, with $\alpha_{s}=0, m_{u}=m_{d}=0, m_{s}=100 \mathrm{MeV}$ and the GM3 hadronic model [5].

zero of the pressure. In particular, in the curve with $B^{1 / 4}=160 \mathrm{MeV}$, the second zero of the pressure corresponds to a second minimum of the energy per baryon number at finite baryon density $\left(\rho=0.274 \mathrm{fm}^{-3}\right)$ in the quarkhadron mixed phase $(\chi=0.55)$. $\square$ There is therefore a range of quark model parameters for which it is possible to realize stable hybrid stars with a sharp surface composed by hadrons and quarks.

The previous analysis is based on the use of Gibbs conditions, which for $\beta$ stable matter are not equivalent to the so-called Maxwell construction. The latter would actually not be possible when the minimum of the quark EOS corresponds to an energy per baryon number smaller than the nucleon mass. Gibbs construction is at the contrary still possible, and reduces the energy of the system in the mixed phase.

It is interesting to note that a very similar behavior of EOS has been recently obtained in Ref. [7] studying phase transition to hyperonic matter in neutron stars. Increasing the strengths of the hyperon-hyperon interactions, the authors find a second minimum of the energy per baryon (i.e. a second

1 For consistency, we have checked that for all the hadron/quark models we have considered, the phase transition from the hadronic matter to the hadron-quark mixed phase in (non-strange) symmetric nuclear matter occurs at baryon densities much larger than the ordinary nuclear matter density. 

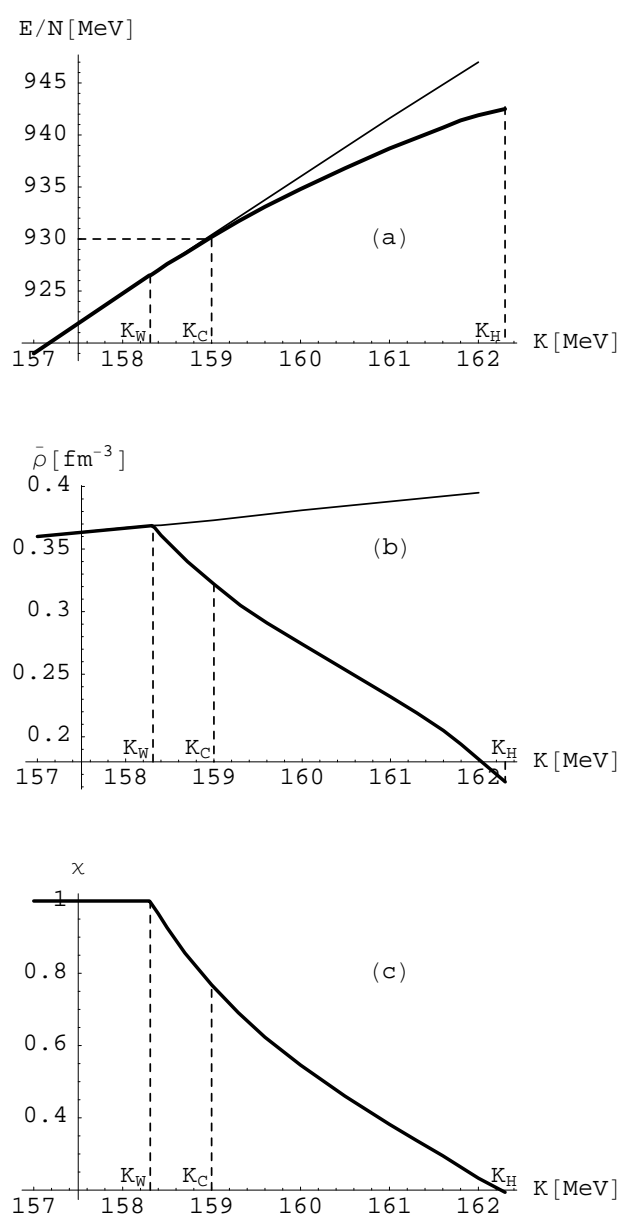

Fig. 2. (a) The energy per baryon number $E / N$ at its second minimum as a function of $K \equiv B^{1 / 4}[\mathrm{MeV}]$; (b) the corresponding baryon density $\bar{\rho}$; (c) the associate fraction of quark matter $\chi$. In Fig. 2a and $2 \mathrm{~b}$ the thin line corresponds to a pure quark EOS (MIT bag model with $\alpha_{s}=0, m_{u}=m_{d}=0, m_{s}=100 \mathrm{MeV}$ ) while the thick line corresponds to matching the quark and the hadronic EOS using Gibbs conditions.

zero of the pressure) at a large strangeness fraction. Such minimum can be deeper than ordinary matter (absolutely stable strange hadronic matter and, consequently, self-bound neutron stars) or not (gravitationally bound stars). Analogous results are obtained by us in the context of the quark-hadron phase transition.

To understand better our results, we show in Fig. 2: (a) the values of the second minimum of the energy per baryon number $E / N$; (b) the corresponding baryon density $\bar{\rho}$ and (c) the associate fraction of quark matter $\chi$, for different 
$K_{i} \equiv B_{i}^{1 / 4}[\mathrm{MeV}]$. In Fig. 2a and $2 \mathrm{~b}$ the thin line represents the results obtained using a pure quark EOS (MIT model, with $\alpha_{s}=0, m_{u}=m_{d}=0, m_{s}=100$ $\mathrm{MeV}$, the dependence of our results on $\alpha_{s}$ and $m_{s}$ will be discussed below) while the thick line corresponds to matching the quark and the hadronic EOS using the Gibbs conditions. For values of the bag constant smaller than $K_{W}$, we have self-bound purely quark stars: the second minimum of the energy per baryon number lies in the pure quark phase. By increasing $K$, we have stars with an increasing fraction of hadronic matter at the surface $(\chi<1)$. In the range between $K_{W}$ and $K_{C}$ the energy per particle is less then $930 \mathrm{MeV}$ and the Bodmer-Witten condition is satisfied: we have therefore self-bound stars with a surface composed by a quark-hadron mixed phase. For values $K_{C}<K<K_{H}$, we have gravitationally bound stars with a sharp edge, while, for $K>K_{H}$, we obtain hybrid stars with a density profile vanishing to zero.

Since we have introduced in the previous paragraph a class of not self-bound stars, it is natural to address the question of their stability. In particular it is important to check that low-mass stars are stable against "evaporation" into iron nuclei. A sufficient condition is the following:

$$
\frac{\partial M}{\partial N}<E_{F e},
$$

where $M$ is the gravitational mass of the star and $N$ is the baryon number. We have checked numerically that, in all models we have considered, stars having a radius larger than $4 \mathrm{~km}$ (or equivalently $M \gtrsim 0.1 M_{\odot}$ ) are stable. Stars for which observational constraints on the $M-R$ relation has been discussed in the literature should have a mass larger than $1 M_{\odot}$. Therefore we will not discuss in detail the low mass region.

In Table 1, we report the results for different hadronic $[5,19]$ and quark models, like the MIT-bag model $\left(K_{i} \equiv B_{i}^{1 / 4}[\mathrm{MeV}]\right)$ and CDM model $\left(K_{i} \equiv g_{i}[\mathrm{MeV}]\right.$ and $M=1.7 \mathrm{GeV}) . K_{\text {Min }}$ is the minimum $K$ for which non-strange quark matter is unbound (the energy per baryon of non-strange quark matter must exceed the lowest energy per baryon in nuclei, i.e., about $930 \mathrm{MeV}$ for iron). We observe, from the third column of the table $\left(K_{W}\right.$ and $\left.E / N\right)$, that only for specific values of quark model parameters it is possible to realize absolutely stable self-bound matter in mixed phase $(E / N \leq 930 \mathrm{MeV})$. For large values of $\alpha_{s}$ and $m_{s}$ the Bodmer-Witten hypothesis is satisfied only in pure quark matter (in other words: $K_{C}<K_{W}$ ). Let us note that the values of the quark model parameters used in the present calculation are close to the values adopted in hadronic calculations (for example, the coupling $g \approx 22 \mathrm{MeV}$ in the CDM is very near to the value $g=23 \mathrm{MeV}$ used to reproduce the experimental mass and radius of the nucleon [22]).

The stable solutions of the Tolman-Oppenheimer-Volkoff equation are shown 


\begin{tabular}{cccc}
\hline \hline Models & $K_{M i n}$ & $K_{W}, E / N$ & $K_{H}, E / N, \chi$ \\
\hline GM1-MIT, $\alpha_{s}=0, m_{s}=100 \mathrm{MeV}$ & 145 & $158.4,927$ & $162.5,943,0.16$ \\
GM3-MIT, $\alpha_{s}=0, m_{s}=100 \mathrm{MeV}$ & 145 & $158.3,927$ & $162.3,943,0.17$ \\
B91 -MIT, $\alpha_{s}=0, m_{s}=100 \mathrm{MeV}$ & 145 & $159.2,931$ & $161.8,942,0.13$ \\
\hline GM1-MIT, $\alpha_{s}=0, m_{s}=150 \mathrm{MeV}$ & 145 & $155.1,931$ & $158.1,943,0.20$ \\
GM3-MIT, $\alpha_{s}=0, m_{s}=150 \mathrm{MeV}$ & 145 & $155.0,930$ & $157.9,943,0.18$ \\
B91 -MIT, $\alpha_{s}=0, m_{s}=150 \mathrm{MeV}$ & 145 & $155.9,935$ & $157.4,942,0.22$ \\
\hline GM1-MIT, $\alpha_{s}=0.3, m_{s}=100 \mathrm{MeV}$ & 137.5 & $150.7,929$ & $154.2,943,0.25$ \\
GM3-MIT, $\alpha_{s}=0.3, m_{s}=100 \mathrm{MeV}$ & 137.5 & $150.6,929$ & $154.0,943,0.23$ \\
B91 -MIT, $\alpha_{s}=0.3, m_{s}=100 \mathrm{MeV}$ & 137.5 & $151.4,934$ & $153.4,943,0.23$ \\
\hline GM1-MIT, $\alpha_{s}=0.3, m_{s}=150 \mathrm{MeV}$ & 137.5 & $147.4,932$ & $150.2,944,0.25$ \\
GM3-MIT, $\alpha_{s}=0.3, m_{s}=150 \mathrm{MeV}$ & 137.5 & $147.4,931$ & $150.0,943,0.27$ \\
B91 -MIT, $\alpha_{s}=0.3, m_{s}=150 \mathrm{MeV}$ & 137.5 & $148.2,936$ & $149.5,942,0.27$ \\
\hline GM3-MIT, $\alpha_{s}=0, m_{s}=200 \mathrm{MeV}$ & 145 & $151.6,936$ & $153.1,943,0.31$ \\
GM3-MIT, $\alpha_{s}=0.3, m_{s}=200 \mathrm{MeV}$ & 137.5 & $143.9,935$ & $145.6,943,0.29$ \\
GM3-MIT $, \alpha_{s}=0.6, m_{s}=200 \mathrm{MeV}$ & 128.1 & $134.8,934$ & $136.7,943,0.33$ \\
\hline GM1-CDM & 19.8 & $21.9,932$ & $23.2,944,0.19$ \\
GM3-CDM & 19.8 & $21.9,932$ & $23.1,942,0.22$ \\
\hline \hline
\end{tabular}

Table 1

Critical values $K_{M i n}, K_{W}$ and $K_{H}$ of the quark models parameter $K\left(K \equiv B^{1 / 4}\right.$ $[\mathrm{MeV}]$, for MIT-bag model and $K \equiv g[\mathrm{MeV}]$, for CDM model). $K_{M i n}$ is the minimum $K$ for which the energy of non-strange quark matter is above $E_{F e}$. For $K<K_{W}$ we have pure quark stars, for $K_{W}<K<K_{H}$ we have hybrid stars with a surface made of quark-hadron mixed phase, for $K>K_{H}$ we have normal hybrid stars with hadronic matter density vanishing at the surface. $E / N[\mathrm{MeV}]$ is the energy per baryon number at the minimum (both for $K=K_{W}$ and for $K=K_{H}$ ), $\chi$ is the fraction of mixed phase made of quark matter.

in Figs. 3 (mass-radius relation) and 4 (energy density profile at fixed gravitational mass $\left.M=1.4 M_{\odot}\right)$. In both figures the curve labeled with GM3 corresponds to the solution obtained using the hadronic EOS alone (no phase transition), while the others are labeled with the adopted value of $B^{1 / 4}[\mathrm{MeV}]$ of the MIT-bag model $\left(\alpha_{s}=0, m_{s}=100 \mathrm{MeV}\right)$. The curves GM3 and $B^{1 / 4}=170 \mathrm{MeV}$ are ordinary neutron stars with hadronic density vanishing at the surface (for subnuclear densities we have employed the Baym-Pethick- 


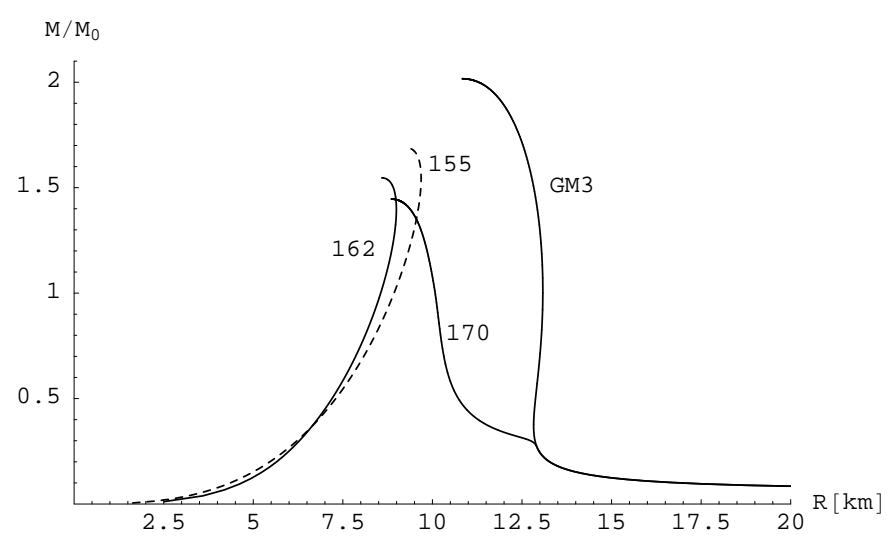

Fig. 3. The mass-radius relation for different EOSs. The curves GM3 and $B^{1 / 4}=170$ $\mathrm{MeV}$ are ordinary neutron stars with a vanishing hadronic density at the surface. The solutions with $B^{1 / 4}=155 \mathrm{MeV}$ are pure quark stars while $B^{1 / 4}=162 \mathrm{MeV}$ corresponds to hybrid stars with a quark matter core and a surface composed of mixed phase $(\chi=0.23)$.

Sutherland EOS [23], which describes the crust of the neutron star). The other two curves are crustless stars with a non-zero density at the surface. The solution with $B^{1 / 4}=155 \mathrm{MeV}$ corresponds to pure quark stars while the one with $B^{1 / 4}=162 \mathrm{MeV}$ represents hybrid stars with a central quark matter core and a surface composed of mixed phase $(\chi=0.23)$. Similar behaviors are obtained by using the CDM.

In the present letter, we have not considered the possibility of having a thin crust of nuclear material, suspended from contact with the high density mixed phase by an electric dipole field [11,17]. The introduction of this thin crust would modify significantly the $M-R$ relationship only for extremely small values of the mass $\left(M \approx 0.1 M_{\odot}\right)[7]$. As discussed above, we are not interested in this range of low mass stars.

The main result of our work is the indication of the possibility to have stars whose surface is composed of a mixed phase of quarks and hadrons. For certain ranges of model parameters, these solutions of the Tolman-OppenheimerVolkoff equation can be obtained without the need to satisfy the BodmerWitten hypothesis and corresponds to gravitationally bound stars. In other parameter ranges, it is possible to satisfy Bodmer-Witten hypothesis in mixed phase and self bound stars are obtained. We stress again that the present analysis could not be done using the Maxwell construction instead of Gibbs conditions, since no Maxwell construction would be possible for a quarks' EOS 


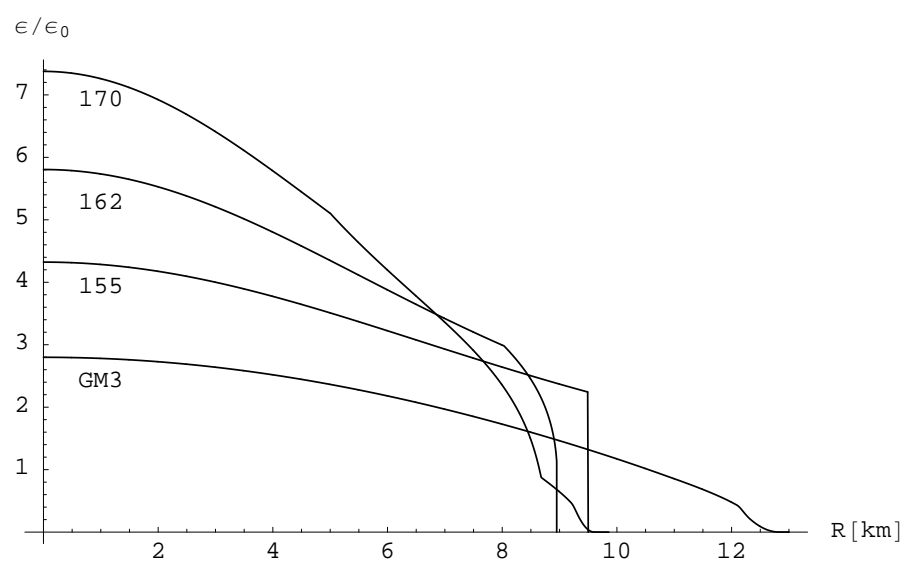

Fig. 4. Energy density profile (in units of the nuclear matter energy density at saturation $\epsilon_{0}$ ) at fixed stellar gravitational mass $M=1.4 M_{\odot}$. Labels as in Fig. 3 .

having a minimum of the energy per baryon number below the mass of the nucleon.

The existence of mixed phase at the surface of a compact star could be crucial in the explanation of the glitches, since in the mixed phase a crystalline structure can develop once the surface tension is taken into account. Moreover a large fraction of hadronic matter in the surface of the star seems to be needed at the light of recent works on r-mode instability [14] and on the cooling of quark stars $[24,25]$.

It is a pleasure to thank $\mathrm{V}$. Barone for a careful reading of the manuscript. This work is supported in part by MURST.

\section{References}

[1] X.-D. Li et al., Phys. Rev. Lett. 83 (1999) 3776; M. Dey et al., Phys. Lett. B 438 (1998) 123.

[2] D.B. Kaplan and A.E. Nelson, Phys. Lett. B 175 (1986) 57; 179 (1986) 409.

[3] V. Thorsson, M. Prakash and J.M. Lattimer, Nucl. Phys. A 572 (1994) 693.

[4] N.K. Glendenning, J. Schaffner-Bielich, Phys. Rev. Lett. 81 (1999) 4564; Phys. Rev. C 60, 025803.

[5] N.K. Glendenning, S.A. Moszkowski, Phys. Rev. Lett. 67 (1991) 2414. 
[6] J. Schaffner, I.N. Mishustin, Phys. Rev. C 53 (1996) 1416.

[7] J. Schaffner-Bielich, M. Hanauske, H. Stöcker, W. Greiner, astro-ph/0005490.

[8] A.R. Bodmer, Phys. Rev. D 4 (1971) 1601.

[9] E. Witten, Phys. Rev. D 30 (1984) 272.

[10] E. Farhi and R.L. Jaffe, Phys. Rev. D 30 (1984) 2379.

[11] C. Alcock, E. Fahri, A. Olinto, ApJ. 310 (1986) 261.

[12] P. Haensel, J.L. Zdunik, R. Schaeffer, Astron. Astrophys. 160 (1986) 121.

[13] N.K. Glendenning, Mod. Phys. Lett. A 5 (1990) 2197.

[14] J. Madsen, Phys. Rev. Lett. 81 (1998) 3311; Phys. Rev. Lett. 85 (2000) 10.

[15] M. Alford, J. Bowers, K. Rajagopal, J. Phys. G 27 (2001) 541; hep-ph/0008208, Phys. Rev. D, in press.

[16] N.K. Glendenning, Phys. Rev. D 46 (1992) 1274;

[17] N.K. Glendenning, Compact Stars, 1997 Springer-Verlag, New York.

[18] K. Schertler, C. Greiner, J. Schaffner-Bielich, M.H. Thoma, Nucl. Phys. A 677 (2000) 463.

[19] A.R. Bodmer, Nucl. Phys. A 526 (1991) 703.

[20] H.J. Pirner, Prog. Part. Nucl. Phys. 29 (1992) 33.

[21] M.C. Birse, Prog. Part. Nucl. Phys. 25 (1990) 1; J.A. McGoven, Nucl. Phys. A 533 (1991) 553.

[22] T. Neuber, M. Fiolhais, K. Goeke, J.N. Urbano, Nucl. Phys. A 560 (1993) 509.

[23] G. Baym, C.J. Pethick, P. Sutherland, ApJ. 170 (1971) 299; G. Baym, H.A. Bethe, C.P. Pethick, Nucl. Phys. A 175 (1971) 225.

[24] D. Blaschke, T. Klähn, D.N. Voskresensky, ApJ. 533 (2000) 406.

[25] A.W. Steiner, M. Prakash, J.M. Lattimer, Phys. Lett. B 486 (2000) 239 and astro-ph/0101566. 